\title{
Lopinavir/Ritonavir in the Treatment of COVID- 19: A Systematic Review and Meta-Analysis
}

\author{
Yan $\mathrm{Du}^{1}$, Tongtong Wang'2, Bo $\mathrm{Li}^{3}$, Fang Pang ${ }^{4}$, Xuanguo Zhang5, $\mathrm{Li} \mathrm{Xi}^{6 *}$ \\ ${ }^{1}$ Jiayuguan Health Commission, Jiayuguan 735100, China \\ ${ }^{2}$ Infectious Diseases Department, Shaanxi Traditional Chinese Medicine Hospital, Xi'an 710003, China \\ ${ }^{3}$ Department of Pharmacy, Shaanxi Traditional Chinese Medicine Hospital, Xi'an 710003, China \\ ${ }^{4}$ College of Traditional Chinese Medicine, Chongqing Medical University, Chongqing 400016, China \\ ${ }^{5}$ Emergency Department and Intensive Care Unit, Shaanxi Traditional Chinese Medicine Hospital, Xi'an 710003, China \\ ${ }^{6}$ Editorial Department of Shaanxi Journal of Traditional Chinese Medicine, Shaanxi Academy of Traditional Chinese \\ Medicine, Xi'an 710003, China
}

*Corresponding author: Li Xi, xili0613@ sina.com

\begin{abstract}
Objective: To systematically evaluate the efficacy and safety of lopinavir/ritonavir (LPV/r) in the treatment of COVID-19. Methods: PubMed, Embase, Ovid, CNKI, CBM, Wanfang, and VIP databases were searched to obtain the clinical studies of LPV/r in the treatment of COVID-19 from December 2019 to July 2020. The literatures were screened according to the inclusion and exclusion criteria. Their qualities were evaluated according to the Newcastle-Ottawa Scale (NOS) and RevMan 5.3 software was used for meta-analysis. Results: A total of 688 patients were included in five studies, involving China and France. Compared with patients in the control group, who was only treated with routine treatment, there were no significant differences of the 7-day nucleic acid negative conversion rate and 14-day nucleic acid negative conversion rate in the treatment group. However, the use of LPV/r increased the incidence of adverse reactions in the treatment group compared to the control group. Conclusion: There is no available evidence to support the use of Lopinavir/ritonavir in the treatment of COVID-19.
\end{abstract}

Keywords: Lopinavir/ritonavir; COVID-19; SARS-CoV-2; Systematic review; Meta-analysis

Publication date: September 2021; Online publication: September 30, 2021

\section{Introduction}

COVID-19 is a public health emergency of international concern. According to the official website of the World Health Organization, as of 1600 Beijing time on July 8, 2020, there were a total of 11,822,959 people infected with COVID-19 and 544,245 deaths worldwide. Since the disease involves SARS-COV-2 infection, antiviral therapy is extremely important. However, there is still no effective antiviral therapy up to now. Lopinavir/ritonavir (LPV/r) is a compound preparation for the treatment of HIV. Previous studies on coronavirus ${ }^{[1]}$ have reported that LPV/r improves the prognosis of SARS and MERS infection, and it can be used in the treatment of COVID-19. Through systematic review and meta-analysis of the clinical studies of LPV/r in the treatment of COVID-19, this article seeks for clinical evidence of the use of LPV/r in the treatment of COVID-19. 


\section{Materials and methods}

\subsection{Inclusion and exclusion criteria}

\subsubsection{Type of studies}

Clinical reports on the use of antiviral therapy, LPV/r, in patients with COVID-19 (including randomized controlled trials, non-randomized controlled trials, retrospective studies, etc.).

\subsubsection{Research subjects}

Patients that were diagnosed with COVID-19 according to the COVID-19 diagnostic guidelines published by various countries.

\subsubsection{Intervention}

The treatment group include patients treated with LPV/r and combined use of routine treatment measures. The control group include patients who are not treated with LPV/r but the routine treatment measures are the same as the treatment group.

\subsubsection{Observation indicators}

The negative conversion rate of virus and the adverse reactions to the medication.

\subsubsection{Exclusion criteria}

Special populations (children, elderly, pregnant women, and patients with specific diseases) and literatures of which the data on the efficacy of LPV/r after treatment could not be obtained from the reported content.

\subsection{Literature retrieval}

PubMed, Embase, Ovid, CNKI, CBM, Wanfang, and VIP databases were searched. Published clinical reports about LPV/r treatment for COVID-19 from December 2019 to July 2020 were selected. The keywords used were "Lopinavir," "Covid-19," and so on. The retrieval method of combining subject words and free words is adopted. Taking PubMed as an example, the specific retrieval strategy is as follows:

(1) "Lopinavir/ritonavir" or "Lopinavir" or "LPV/r" or "Lopinavir plus Ritonavir";

(2) "2019 novel coronavirus disease" or "COVID19" or "COVID-19 pandemic" or "SARS-CoV-2 infection" or "COVID-19 virus disease" or "2019 novel coronavirus infection" or "2019-nCoV infection" or "coronavirus disease 2019" or "coronavirus disease-19" or "2019-nCoV disease" or "COVID-19 virus infection" or "COVID-19";

(3) (1) and (2).

\subsection{Literature screening and data extraction}

Two researchers independently screened the literatures, extracted the data, and conducted cross-checking. In cases where there were differences, a third researcher was consulted to assist in judging. Specific authors of the literatures were contacted to supplement the lack of data as much as possible. When screening the literatures, the titles and abstracts were first read, and after excluding irrelevant literatures, the full text of each literature was read to determine whether it should be included or not. The main contents of the data extracted were the author, region and time, number of study samples, gender composition of patients, intervention measures, and observation indicators. 


\subsection{Quality evaluation}

Considering the large number of patients and the high contagion of the disease, it is difficult to adopt placebo control, randomization, blinding, and allocation concealment; thus, the existing studies were based on retrospective studies. Therefore, the quality of the included literatures were evaluated according to the Newcastle-Ottawa Scale (NOS) ${ }^{[2]}$. The semi-quantitative principle of the star system was adopted with a full score of 9. The literatures were evaluated using eight items in three parts, which include selection, comparability, as well as exposure and outcome.

\subsection{Statistical analysis}

The literature data that can be combined with effects for meta-analysis were analyzed by RevMan 5.3 software. The weighted mean difference (MD) was used as the effect size in the quantitative data while the relative risk (RR) was used as the effect size in the qualitative data. The point estimate and 95\% CI of each effect were given. The heterogeneity among the results was analyzed by $\chi^{2}$ test, and the heterogeneity was quantitatively judged by $\mathrm{I}^{2}$. If the heterogeneity of the results is small, the fixed effect model combined with the effect for meta-analysis is used whereas if there is a large statistical heterogeneity between the results, the random effect model with the effect for meta-analysis is used.

\section{Results}

\subsection{Document screening process and results}

One thousand three hundred and thirty five related studies were initially detected, and after layer-by-layer screening, only 5 studies were included ${ }^{[3-7]}$, and 4 studies ${ }^{[3-6]}$ can be studied by meta-analysis. For the other literatures, there were either significant differences in intervention measures and outcomes, or the description of outcomes cannot be studied by meta-analysis, but only through descriptive analysis. The literature screening process and results are shown in Figure 1.

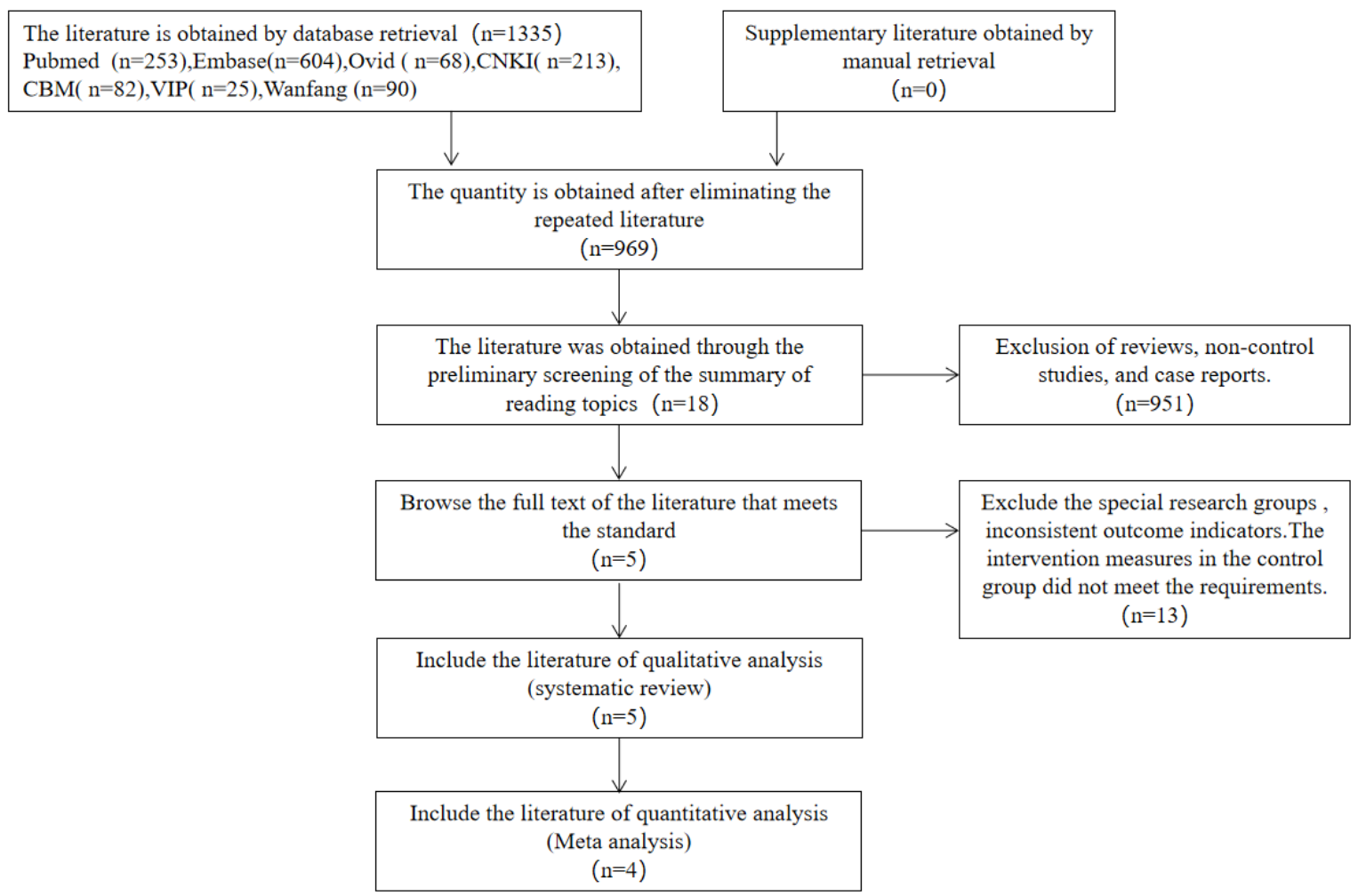

Figure 1. Flow chart of document retrieval 


\subsection{Basic characteristics and the quality evaluation of the included studies}

The basic characteristics of the included studies are shown in Table 1. A total of five studies were included, involving China and France, with a total of 688 patients, where 339 of them were male patients and 349 were female patients. The patients were mainly treated with LPV/r, including arbidol, hydroxychloroquine, ribavirin, interferons, Lianhua Qingwen capsules, glucocorticoids, antibiotics, immunoglobulin, thymosin, and other adjuvant therapies. Four studies ${ }^{[3-5,7]}$ were retrospective studies, and one study ${ }^{[6]}$ was a prospective real-world observational study. Four studies ${ }^{[3-4,6-7]}$ indicated that they had passed the review and informed consent of the ethics committee, but one study ${ }^{[5]}$ did not mention it.

Two researchers independently evaluated the quality of the included literatures and provided the star semi-quantitative score by means of a scoring system. For controversial cases, a third researcher was appointed to vote by 3 people. The scores of the five studies were between 5 to 7 , of which most of them were 6 points and of medium quality.

Table 1. Basic characteristics of the included studies

\begin{tabular}{|c|c|c|c|c|c|c|c|}
\hline \multirow{2}{*}{$\begin{array}{l}\text { Author and time } \\
\text { of publication }\end{array}$} & \multirow{2}{*}{ Country/region } & \multirow{2}{*}{$\begin{array}{l}\text { Sample } \\
\text { size }\end{array}$} & \multirow{2}{*}{$\begin{array}{c}\text { Sex } \\
\text { (Male/Female) }\end{array}$} & \multicolumn{2}{|c|}{ Intervention } & \multirow{2}{*}{$\begin{array}{l}\text { Observation } \\
\text { indicator }\end{array}$} & \multirow[b]{2}{*}{ NOS } \\
\hline & & & & $\begin{array}{l}\text { Treatment } \\
\text { group }\end{array}$ & Control group & & \\
\hline $\begin{array}{l}\text { Wen, et al. }{ }^{[3]} \\
\text { May 9, } 2020\end{array}$ & $\begin{array}{l}\text { Guangzhou, } \\
\text { China }\end{array}$ & 178 & $81 / 97$ & $1+2$ & $\begin{array}{c}\text { A: } 1 \quad \text { B: } 1+3 \\
\text { C: } 1+2+3\end{array}$ & (1) (2) & 6 \\
\hline $\begin{array}{c}\text { Yu, et al. }{ }^{[4]} \\
\text { March 10, } 2020\end{array}$ & $\begin{array}{l}\text { Wuhan, } \\
\text { China }\end{array}$ & 222 & $105 / 117$ & $1+2$ & 1 & (2) & 6 \\
\hline $\begin{array}{l}\text { Chen, et al. }{ }^{[5]} \\
\text { February } 2020\end{array}$ & $\begin{array}{l}\text { Shanghai, } \\
\text { China }\end{array}$ & 134 & $69 / 65$ & $1+2$ & A: $1 \quad$ B: $1+3$ & (1) (2) & 5 \\
\hline $\begin{array}{l}\text { Wang, et al. }{ }^{[6]} \\
\text { July } 8,2020\end{array}$ & $\begin{array}{l}\text { Wuhan, } \\
\text { China }\end{array}$ & 109 & $49 / 60$ & $1+2$ & 1 & (2) & 7 \\
\hline $\begin{array}{l}\text { Sami, et al. }{ }^{[7]} \\
\text { May 24, } 2020\end{array}$ & France & 45 & $35 / 10$ & 2 & A: $1 \quad$ B: 4 & (1) & 6 \\
\hline
\end{tabular}

Note: 1: Routine treatment (isolation, nursing, bed rest, symptomatic and supportive treatment, antiviral, antibacterial, glucocorticoid shock, oxygen therapy, or auxiliary breathing according to the needs of the disease); 2: LPV/r; 3: Arbidol; 4: Hydroxychloroquine; (1): Negative conversion rate by PCR; (2): Incidence of adverse reactions

\subsection{Meta-analysis of main observation indicators}

\subsubsection{Negative conversion rate of viral nucleic acid}

There were 3 studies ${ }^{[3,5,6]}$ that reported the results of negative conversion rate of viral nucleic acid after 7 days of treatment. The heterogeneity test, $\mathrm{I}^{2}=68 \%$, indicated that the three studies had some heterogeneity. Using the random effect model, the results showed that $\mathrm{RR}=0.67,95 \% \mathrm{CI}[0.32,1.40], \mathrm{Z}=1.06$, and $\mathrm{P}=$ 0.29 , indicating that there was no statistically significant difference in the negative conversion rate of viral nucleic acid between the treatment group and the routine treatment group after 7 days of treatment as shown in Figure 2. 


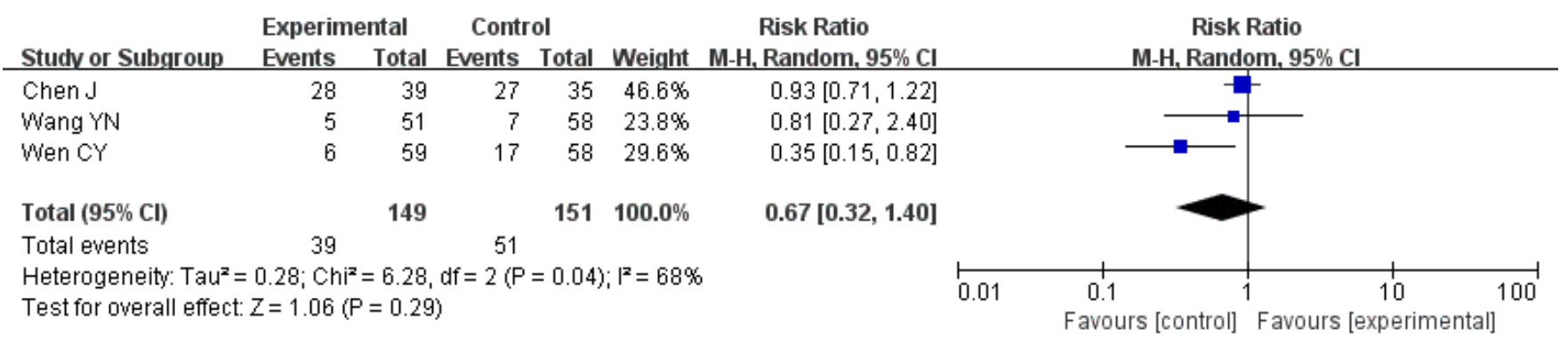

Figure 2. Forest plot for Day-7 nucleic acid negative conversion rate (compared with routine treatment)

There were two studies ${ }^{[3,6]}$ that reported the results of negative conversion rate of nucleic acid after 14 days of treatment, and the heterogeneity test showed that the two studies had some heterogeneity $\left(\mathrm{I}^{2}=50 \%\right)$. The results were analyzed by the random effect model, and the results showed that $\mathrm{RR}=0.87,95 \% \mathrm{CI}$ $[0.55,1.36], \mathrm{Z}=0.63$, and $\mathrm{P}=0.53$, indicating that there was no statistically significant difference in the negative conversion rate of viral nucleic acid between the treatment group and the routine treatment group at the $14^{\text {th }}$ day after treatment as shown in Figure 3.

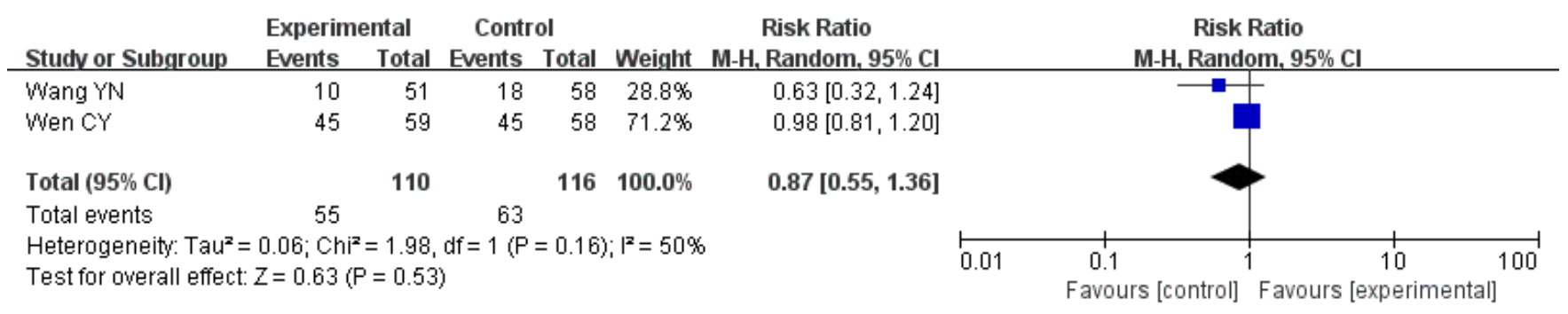

Figure 3. Forest plot for Day-14 nucleic acid negative conversion rate (compared with routine treatment)

One study ${ }^{[7]}$ reported a Day-6 negative conversion rate. There were 5 cases (38\%) in the LPV/r group, 3 cases $(18 \%)$ in the hydroxychloroquine group, and 2 cases $(20 \%)$ in the control group. There were no significant differences among the three groups.

\subsubsection{Incidence of adverse reactions}

There were 4 studies ${ }^{[3-6]}$ that reported the incidence of adverse reactions. The heterogeneity test showed that the homogeneity of the four studies was low $\left(\mathrm{I}^{2}=0 \%\right)$. The results were analyzed by the fixed effect model and showed that $\mathrm{RR}=2.69,95 \% \mathrm{CI}[1.97,3.66], \mathrm{Z}=6.25$, and $\mathrm{P}<0.00001$, indicating that there was a statistically significant difference in the incidence of adverse reactions between the LPV/r group and the routine treatment group, in which the use of LPV/r increased the incidence of adverse reactions as shown in Figure 4.

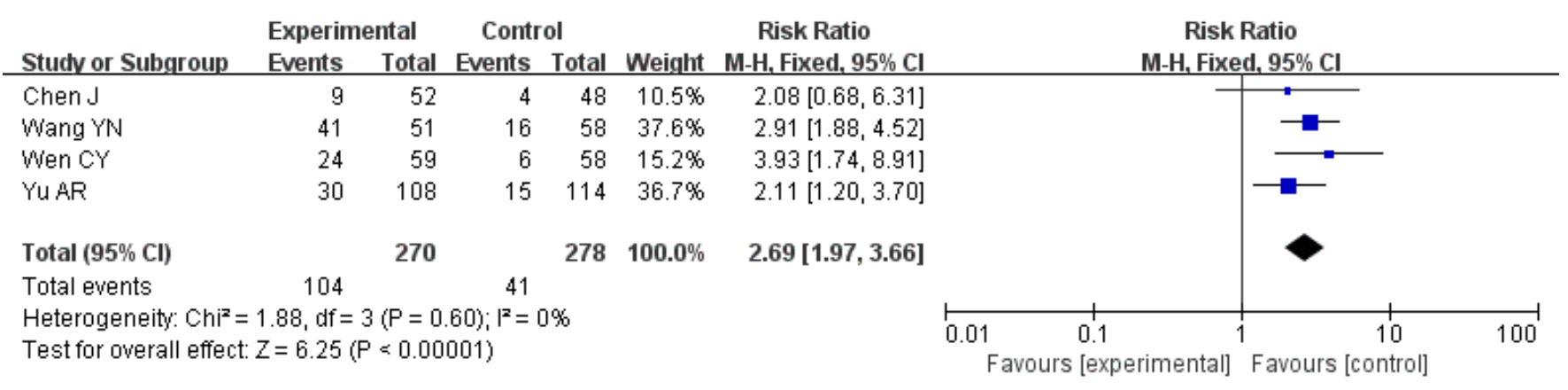

Figure 4. Forest plot for the incidence of adverse reactions (compared with routine treatment) 
The adverse reactions observed in the studies ${ }^{[3-6]}$ were mainly directed at the digestive system, which include diarrhea, nausea, vomiting, loss of appetite, abnormal liver function, anemia, palpitation, gastrointestinal bleeding ${ }^{[6]}$, stomachache, rash with pruritus, and elevated blood sugar ${ }^{[4]}$.

\section{Discussion}

At present, the drug treatment of COVID-19 is still in the exploratory stage. The choice of antiviral drugs needs to be solved urgently. The World Health Organization (WHO) established the Solidarity Trial to look for an effective treatment for hospitalized COVID-19 patients. These interim trial results showed that hydroxychloroquine and LPV/r effectuated minimal or no reduction at all in the mortality of hospitalized COVID-19 patients when compared to standard care ${ }^{[8]}$. For each of the drug, the interim results did not provide solid evidence of increased mortality; however, there were some associated safety signals in the clinical laboratory findings of additional discovery trials of the participants; thus, the Solidarity Trial investigators had suspended the trials with immediate effect. Based on the meta-analysis of existing evidence, there was no significant difference in the nucleic acid negative conversion rate between LPV/r and routine treatment. However, compared with the control group, the use of LPV/r increased the risk of adverse reactions relating to the digestive system, delirium, respiratory failure, and other serious adverse events. Therefore, it is not recommended to use LPV/r for COVID-19.

As most of the included literatures are retrospective studies and lack rigorous randomized, doubleblinded, placebo-controlled trials, there are cases of combined usage of other drugs; thus, the outcome cannot be strictly defined as a result of the use of LPV/r. In regard to that, there is an urgent need for more high-quality data from clinical trials to further confirm. WHO's decision applies only to the conduct of the Solidarity Trial in hospitalized patients; it does not affect the evaluation of other studies of hydroxychloroquine or LPV/r in non-hospitalized patients, or the evaluation for pre- or post-exposure prophylaxis for COVID-19. Therefore, the future of LPV/r in the treatment of COVID-19 still requires further clarification, and whether it is more suitable for pre-exposure or post-exposure prophylaxis, further studies and evaluation are mandatory.

\section{Disclosure statement}

The authors declare that there is no conflict of interest.

\section{References}

[1] Chan FW, Yao Y, Man-Lung Y, et al., 2015, Treatment with Lopinavir/Ritonavir or Interferon- $\beta 1 b$ Improves Outcome of MERS-CoV Infection in a Nonhuman Primate Model of Common Marmoset. Journal of Infectious Diseases, : 1904.

[2] Wells GSB, O'Connell D, 2020, New Castle-Ottawa Quality Assessment Scale - Cohort Studies. http://www.ohri.ca/programs/clinical_epidemiology/oxford.asp (accessed on July 8, 2020).

[3] Wen CY, Xie ZW, Li YP, et al., 2020, Real-World Efficacy and Safety of Lopinavir/Ritonavir and Arbidol in Treating with COVID-19: An Observational Cohort Study. Zhonghua Nei Ke Za Zhi, 59: E012.

[4] Yu A, Fan X, Zhao Y, et al., 2020, Clinical Efficacy and Safety of Lopinavir/ritonavir Combined with Other Antiviral in the Treatment of Coronavirus Disease 2019 (COVID-19). Herald of Medicine, 39(5): 628-632.

[5] Chen J, Ling Y, Xi X, et al., 2020, Efficacies of Lopinavir/Ritonavir and Abidol in the Treatment of Novel Coronavirus Pneumonia. Chin J Infect Dis, 38: 86-89. 
[6] Wang Y, Suo T, Fan H, et al., 2021, Clinical Efficacy of Lopinavir-Ritonavir Combined with Interferon Alpha in COVID-19. Medical Journal of Wuhan University, 42(04): 594-598.

[7] Hraiech S, Bourenne J, Kuteifan K, et al., 2020, Lack of Viral Clearance by the Combination of Hydroxychloroquine and Azithromycin or Lopinavir and Ritonavir in SARS-Cov-2-Related Acute Respiratory Distress Syndrome. Annals of Intensive Care, 10: 63.

[8] World Health Organization, WHO Discontinues Hydroxychloroquine and Lopinavir/Ritonavir Treatment Arms for COVID-19. https:/www.who.int/zh/news-room/detail/04-07-2020-whodiscontinues-hydroxychloroquine-and-lopinavir-ritonavir-treatment-arms-for-covid-19 (accessed on July 8, 2020). 